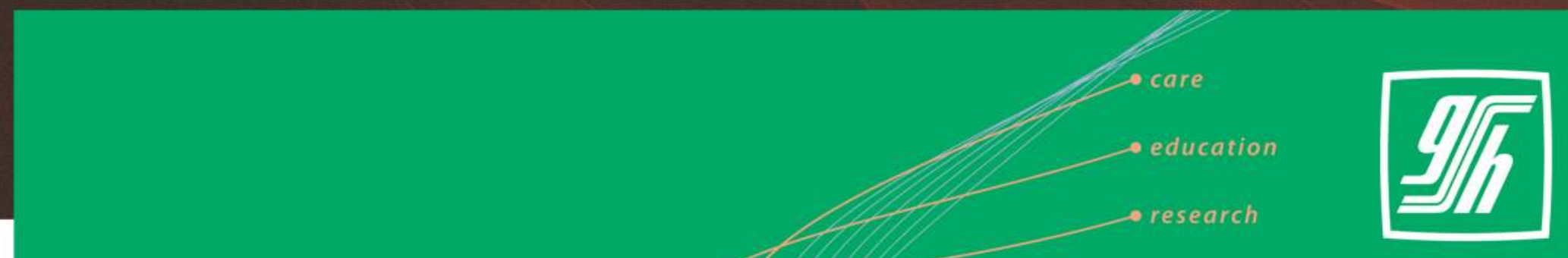

\title{
A Case of Unusual Skin rash - Cutaneous Protothecosis.
}

M.G. $\operatorname{Tan}^{1}$, J.H.C.Sim ${ }^{1}$, M.P. Chlebicki' ${ }^{2}$ B.C. Khoo' 1 L.L. $\operatorname{Tan}^{1}$ \& A.L. $\operatorname{Tan}^{1}$

1 Department of Microbiology, Singapore General Hospital, Singapore ${ }^{2}$ Department of Infectious Diseases, Singapore General Hospital, Singapore

\section{OBJECTIVE}

Cutaneous protothecosis is an unusual cause of skin lesion. It is often misdiagnosed as presentation is similar to many other skin diseases, and cultures are often not performed until much later when there is a lack of clinical improvement. We aim to highlight this unusual pathogen through this case report of cutaneous protothecosis in an elderly diabetic lady.

Patient presented initially with persistent nonpainful erythematous rash on left arm for 6 months. The lesion evolved into multiple umbilicated erythematous firm papules which coalesced

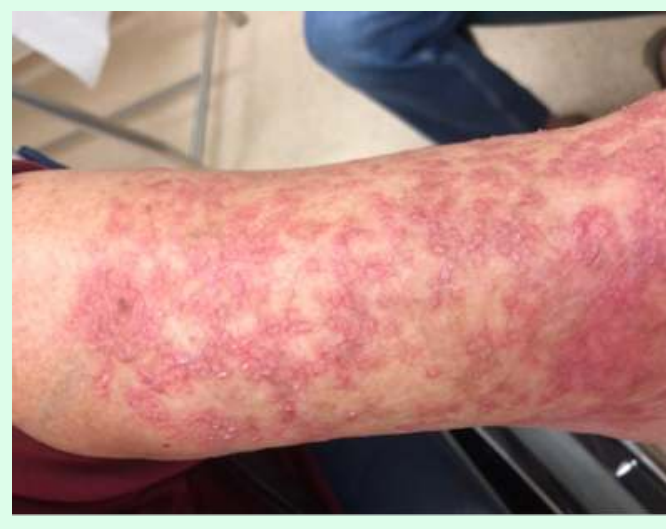

and extended into the left forearm. Differential diagnosis by the dermatologist includes contact dermatitis, elastosis serpinginosa perforans and non-tuberculous mycobacterial infection. Patient was started on topical steroids, and subsequently topical and oral antibiotics with no improvement. A punch biopsy was eventually performed and samples were sent for histological and microbiological examination

\section{METHODS}

Gram-stain was performed on biopsied samples that were sent to the microbiology lab. Specimens were also processed for fungal and bacterial culture using standard microbiological methods. Susceptibility to antifungal was performed using Sensititre YeastOne Y010 (Thermo Scientific) and Etest (bioMérieux) .

\begin{tabular}{|l|l|}
\hline \multicolumn{2}{|c|}{ RESULTS } \\
\hline MICROSCOPY & $\begin{array}{l}\text { Gram-stain: No organism seen } \\
\text { Fungal Microscopy : No fungus seen }\end{array}$ \\
\hline CULTURE & $\begin{array}{l}\text { Bacterial Culture : Pseudomonas } \\
\text { aeruginosa } \\
\text { like-colonies. }\end{array}$ \\
\hline
\end{tabular}

\begin{tabular}{|c|c|c|c|}
\hline $\begin{array}{l}\text { NET MOUNT } \\
\text { Lactophenol Blue } \\
\text { Sotton) }\end{array}$ & \multicolumn{3}{|c|}{ 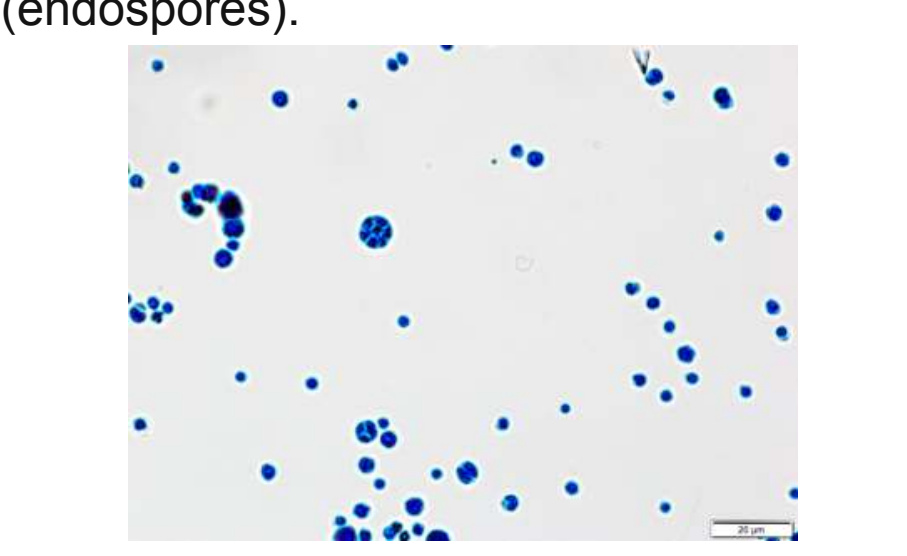 } \\
\hline $\begin{array}{l}\text { API 20C AUX } \\
\text { /itek } 2 \text { (bioMérieux) }\end{array}$ & \multicolumn{3}{|c|}{ Prototheca wickerhamii } \\
\hline \multirow{3}{*}{$\begin{array}{l}\text { Susceptibility test } \\
\text { results } \\
\text { ( } \mu \mathrm{g} / \mathrm{mL})\end{array}$} & & Etest & $\begin{array}{l}\text { Sensititre } \\
\text { Y010 }\end{array}$ \\
\hline & Posaconazole & 0.75 & 1.0 \\
\hline & Amphotericin B & 0.38 & 0.25 \\
\hline istology & \multicolumn{3}{|c|}{$\begin{array}{l}\text { Focal lymphohistiocytic and plasma cell } \\
\text { infiltrate at the superficial dermis with } \\
\text { organisms; spherical-shaped organisms } \\
\text { with occasional morular pattern which } \\
\text { are positive on the Periodic Acid Schiff } \\
\text { (PAS) and Gomori Methenamine Silver } \\
\text { (GMS) stains (see photos), suggestive of } \\
\text { Prototheca species. }\end{array}$} \\
\hline
\end{tabular}

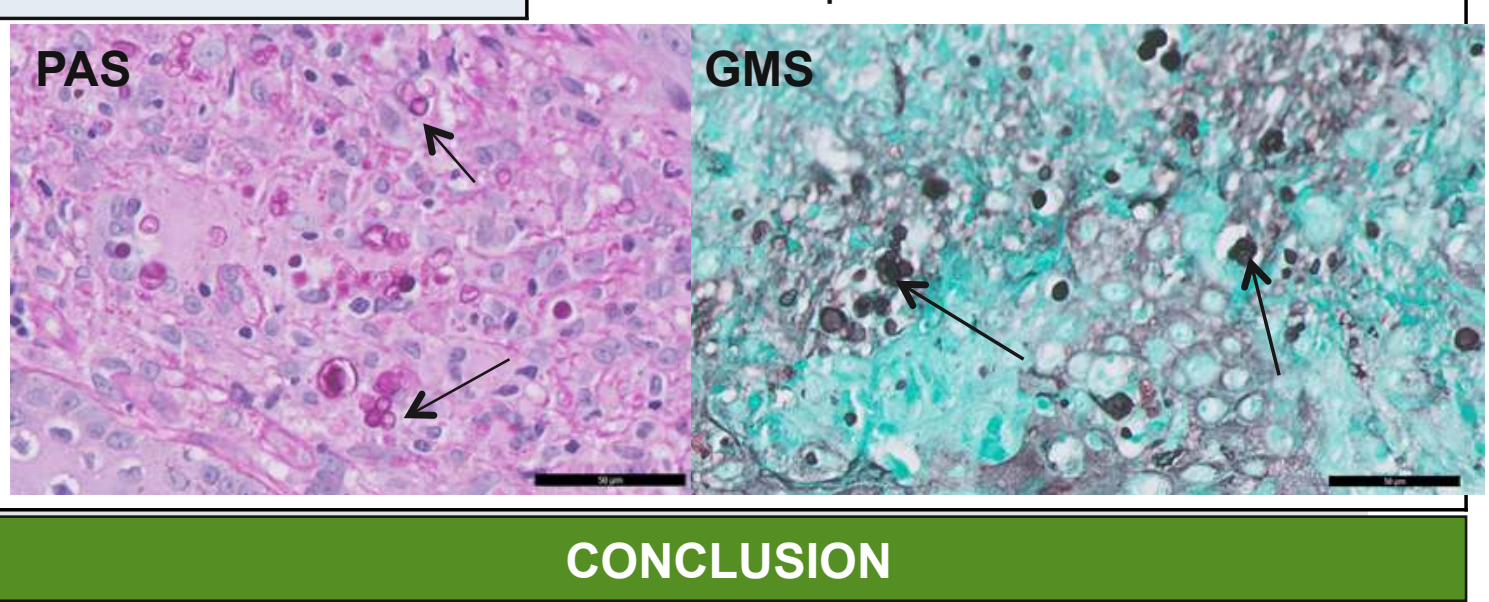

Patient was initially started on oral itraconazole but response was poor. Oral posaconazole was prescribed when susceptibility results were available. Patient recovered well subsequently with resolution of rash.

The diagnosis of Prototheca can be missed as it is a rare cause of skin rash especially in patients with no apparent risk factors. In addition, all yeast-like colonies must be fully identified in order not to miss the diagnosis. Both culture and histology are important, and were used to clinch the diagnosis in this case.Therefore it is important to correlate clinical, microbiology and histology findings to make an accurate diagnosis of protothecosis. 